\title{
Meta
}

Journal des traducteurs

Translators' Journal

\section{Le vocabulaire des fluctuations dans le discours économique : synonymie et combinatoire}

\section{Serge Verlinde}

Volume 42, numéro 1, mars 1997

Lexicologie et terminologie

URI : https://id.erudit.org/iderudit/004560ar

DOI : https://doi.org/10.7202/004560ar

Aller au sommaire du numéro

Éditeur(s)

Les Presses de l'Université de Montréal

ISSN

0026-0452 (imprimé)

1492-1421 (numérique)

Découvrir la revue

Citer cet article

Verlinde, S. (1997). Le vocabulaire des fluctuations dans le discours économique : synonymie et combinatoire. Meta, 42(1), 5-14.

https://doi.org/10.7202/004560ar
Résumé de l'article

L'auteur soumet neuf couples de mots - qui permettent de qualifier les fluctuations de notions économiques et que les dictionnaires présentent comme synonymes -, à une analyse de leur combinatoire dans un important corpus de textes économiques, scientifiques et de vulgarisation (français et belges). L'ensemble des informations recueillies oblige à conclure que la synonymie des mots étudiés n'est que partielle. Les apprenants de français langue étrangère et les traducteurs qui désirent encoder un message ont donc besoin de davantage d'informations que celles données dans les dictionnaires classiques. L'auteur propose dès lors un traitement dictionnairique qui tend à intégrer les résultats de son étude. 


\title{
LE VOCABULAIRE DES FLUCTUATIONS DANS LE DISCOURS ECONOMIQUE: SYNONYMIE ET COMBINATOIRE*
}

\author{
SERGE VERLINDE \\ Instituut voor Levende Talen, K.U. Leuven, Belgique
}

\begin{abstract}
Résumé
L'auteur soumet neuf couples de mots - qui permettent de qualifier les fluctuations de notions économiques et que les dictionnaires présentent comme synonymes -, à une analyse de leur combinatoire dans un important corpus de textes économiques, scientifiques et de vulgarisation (français et belges). L'ensemble des informations recueillies oblige à conclure que la synonymie des mots étudiés n'est que partielle. Les apprenants de français langue étrangère et les traducteurs qui désirent encoder un message ont donc besoin de davantage d' informations que celles données dans les dictionnaires classiques. L'auteur propose dès lors un traitement dictionnairique qui tend à intégrer les résultats de son étude.
\end{abstract}

\begin{abstract}
A corpus of economic, scientific and popularization texts produced in France and Belgium serves as the basis of this study of 9 word combinations used in economic discourse, and considered as synonyms by dictionaries. Findings indicate that these words are but partial synonyms and imply that standard dictionaries need to provide additional information to enhance their utility and effectiveness for language practionners. The author suggests a practical application of these findings to improve dictionary writing.
\end{abstract}

\section{PRÉLIMINAIRES}

L'étude de la combinatoire du vocabulaire des fluctuations dans le discours économique doit être située dans le cadre d'un projet lexicographique consacré au français économique: le Dictionnaire contextuel du français économiquel (DICOFE). Le DICOFE est un dictionnaire d'encodage (de production ou d'apprentissage) destiné à des apprenants de niveau intermédiaire. La particularité essentielle du DICOFE réside dans le fait qu'il intègre les structures (les combinaisons de mots) pertinentes employées pour parler de l'économie dans une introduction fort élémentaire à ce domaine ${ }^{2}$. La macrostructure du dictionnaire est donc de type onomasiologique et l'objectif principal réside dans le fait que nous avons voulu dépasser la simple description terminologique pour offrir aux apprenants des structures immédiatement utilisables lors de l'encodage d'un message.

Parmi ces structures pertinentes figurent sans conteste les combinaisons entre les termes économiques et les mots et expressions qui traduisent des fluctuations («augmentation», «diminution»), très fréquentes en économie.

Un premier problème, très vaste, se situe sur le plan de l'inventaire de ces mots et expressions, particulièrement nombreux. Un deuxième problème provient de leur emploi contextuel exact.

C'est ce deuxième problème que nous voulons aborder dans cette contribution par le biais de l'examen, dans un important corpus de textes économiques (1.), de la combi- 
natoire de 9 couples de mots du vocabulaire des fluctuations (VF) que les dictionnaires généraux, tels le TLF ou le Grand Robert, qualifient de (quasi-)synonymes:

\begin{tabular}{|ll|ll|}
\hline sens «augmenter» & $\begin{array}{l}\text { identification } \\
\text { du couple }\end{array}$ & sens «diminuer» & $\begin{array}{l}\text { identification } \\
\text { du couple }\end{array}$ \\
\hline $\begin{array}{l}\text { (s')accroître/accroissement } \\
\text { augmenter/augmentation } \\
\begin{array}{l}\text { croître/croissance } \\
\text { hausser/hausse }\end{array}\end{array}$ & $\begin{array}{l}\text { accroi- } \\
\text { augment- } \\
\text { progresser/progression }\end{array}$ & $\begin{array}{l}\text { (se)réduire/réduction } \\
\text { diminuer/diminution }\end{array}$ & $\begin{array}{l}\text { rédu- } \\
\text { dimin- }\end{array}$ \\
haus- & progres- & baisser/baisse & bais- \\
régresser/régression & régres- \\
\hline
\end{tabular}

Dans notre corpus, ces 18 mots totalisent 5884 occurrences pour l'un des sens donnés en combinaison avec quelque 504 substantifs et adjectifs différents.

La question posée étant de savoir si le vocabulaire des fluctuations connaît une combinatoire aléatoire ou non avec le vocabulaire économique (2.). Nous terminons notre contribution par un retour à notre point de départ: l'intégration des résultats de notre étude dans une description de type lexicographique ${ }^{3}$ (3.).

\section{LE CORPUS}

Pour notre étude, nous nous sommes basé sur un corpus de textes économiques comprenant 1084498 mots. Ce corpus se scinde en deux composantes bien distinctes. Une première partie du corpus ( 685669 mots) est constituée de textes de vulgarisation économique d'origine belge: des articles de presse provenant des journaux La Libre Belgique et Le Soir, couvrant une période allant de 1986 à $1992^{4}$, une série d'articles parus dans le bulletin hebdomadaire d'une importante banque belge (la Kredietbank) et une synthèse de l'activité économique belge (Herremans, s.d.). Par la suite, nous désignons cette composante du corpus par la lettre P (corpus Presse).

La seconde partie du corpus se compose de textes économiques français à caractère scientifique, fournis avec le didacticiel LECTICIEL, qui a été mis au point par une équipe de l'ENS de Saint-Cloud. Ces textes comptent 398829 mots et datent, à quelques exceptions près, de la fin des années quatre-vingt. Cette partie du corpus est indiquée par la lettre $\mathrm{L}$ (corpus Lecticiel).

Le corpus automatisé se rapporte à deux communautés linguistiques différentes. Il peut être considéré comme représentatif des deux communautés, vu la présence d'un nombre important d'auteurs.

Comme le démontrent les graphiques suivants (graphique 1 pour le sens «augmenter» et graphique 2 pour le sens «diminuer»), les différences d'emploi du VF avec le vocabulaire économique (le mot prix dans l'exemple) dans les deux composantes du corpus ne semblent pas significatives, même s'il existe quelques rares exceptions (Verlinde 1995: 27-66).

Les pourcentages visualisés par le graphique représentent pour les deux sens une comparaison entre corpus de la proportion des combinaisons de chaque couple du VF avec le mot prix. 


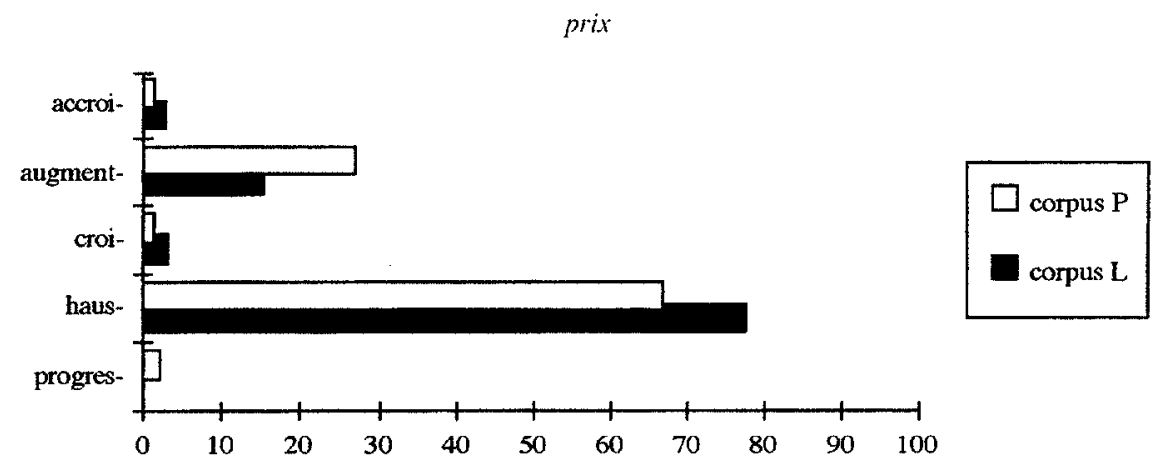

Graphique 1:

Comparaison corpus presse (125 ex.) - corpus Lecticiel (194 ex.): combinaison VF («augmenter») et prix

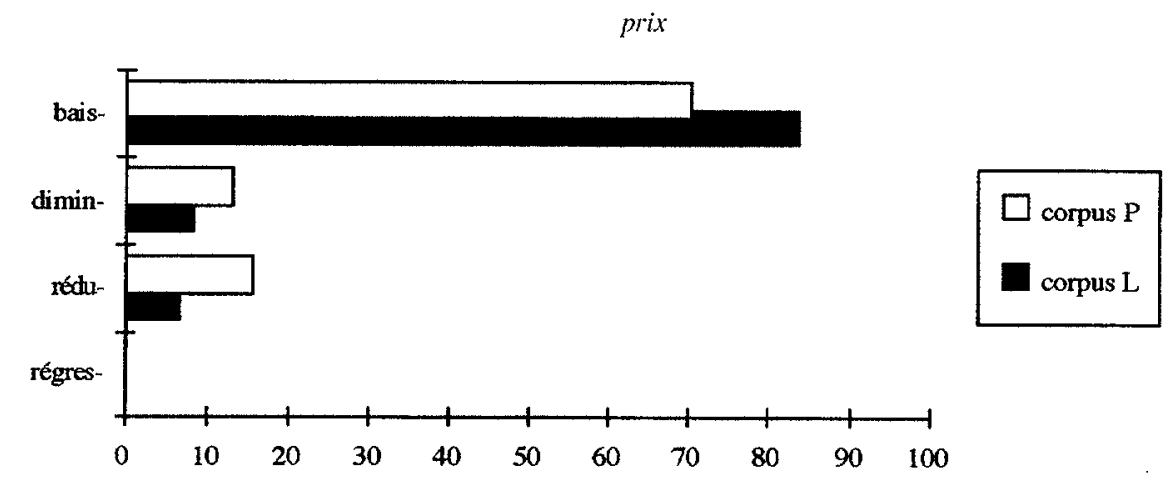

Graphique 2:

Comparaison corpus presse ( 88 ex.) - corpus Lecticiel (56 ex.): combinaison VF («diminuer») et prix

\section{L'ANALYSE DU CORPUS}

2.1. L'EMPLOI DU VF

Une première donnée assez surprenante que nous révèle la radiographie de notre corpus est la disparité d'emploi du verbe et du substantif à l'intérieur de chaque couple du VF à l'étude (graphiques 3 et 4). 


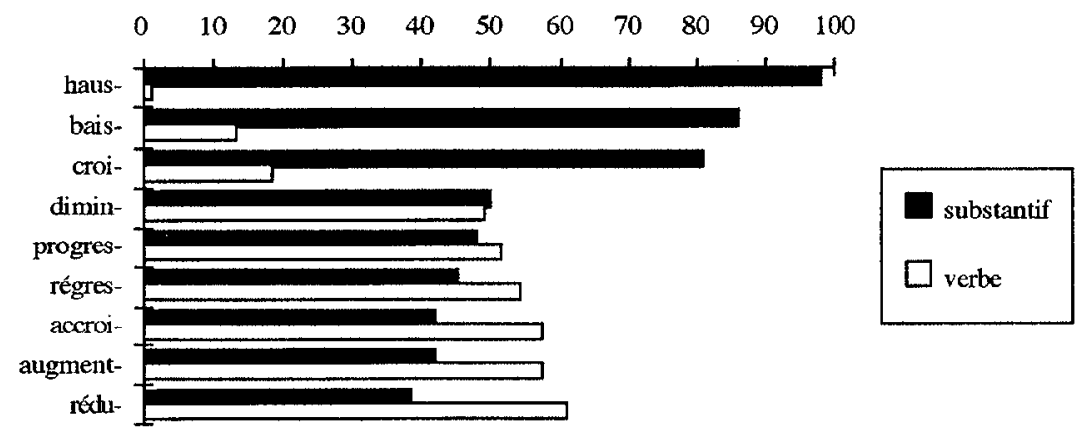

Graphique 3:

Proportion (en pourcentage) de substantifs et de verbes à l'intérieur de chaque couple (corpus presse)

Si la très faible fréquence du verbe hausser par rapport au substantif hausse ne surprend pas, il en est tout autrement pour baisser par rapport à baisse et dans une moindre mesure pour croître par rapport à croissance (à cause de la morphologie du verbe, probablement). Cette tendance se vérifie également dans le corpus français (graphique 4).

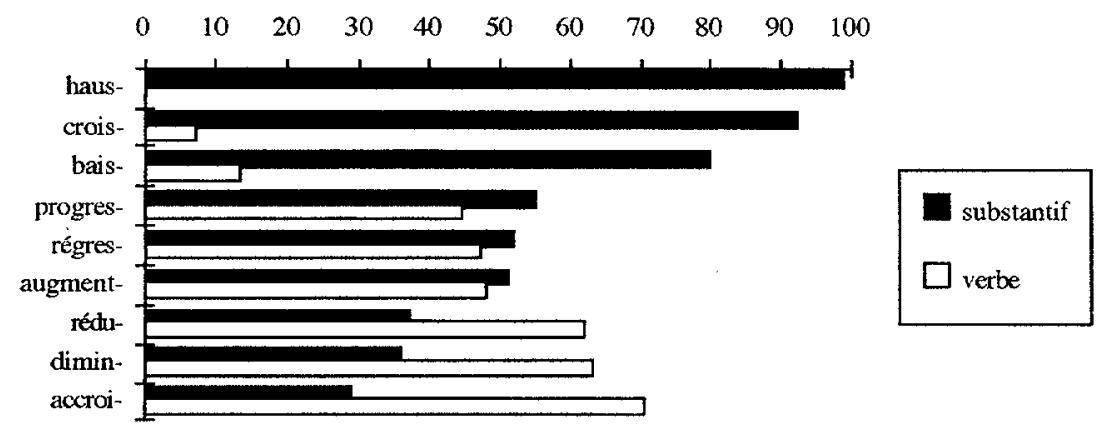

Graphique 4:

Proportion (en pourcentage) de substantifs et de verbes à l'intérieur de chaque couple (corpus Lecticiel) 
Pour les autres couples du VF, l'emploi du verbe semble nettement plus généralisé, d'autant plus que le nombre de verbes est largement inférieur au nombre de substantifs dans un texte.

\subsection{LA COMBINATOIRE DU VF}

Compte tenu du statut de (quasi-)synonymes accordé par les dictionnaires généraux aux couples représentant les sens «augmenter» et «diminuer», on serait tenté de croire que leur combinatoire avec le VF est libre. Cela n'est cependant pas le cas. Même s'il n'est pas possible de parler de combinaisons exclusives comme dans des expressions figées telles qu'augmentation de capital, il existe bel et bien une combinatoire préférentielle entre un bon nombre de termes économiques et l'un des membres du VF que nous étudions.

Un aperçu de la concurrence que se livrent les termes du VF pour entrer en combinaison avec le vocabulaire économique est révélé par les quatre graphiques suivants montrant la combinatoire du VF avec les termes boursiers (graphique 5) et les mots prix (graphique 6), exportation (graphique 7) et nombre (graphique 8) pour l'ensemble du corpus.

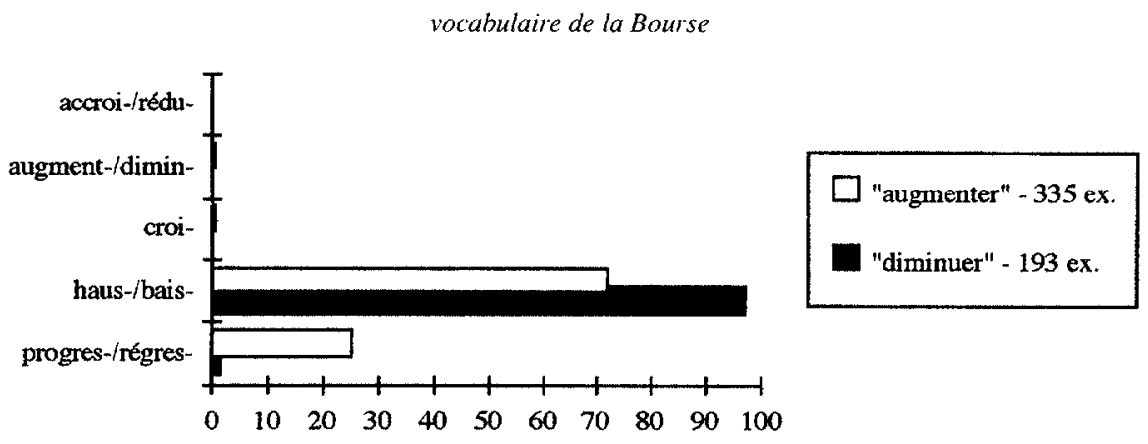

Graphique 5:

Combinaison VF et vocabulaire de la bourse

Le vocabulaire qui se rapporte à la bourse est très fréquent mais très diversifié dans le corpus presse : titre, action, cours, etc.; dénomination du titre (Eurodisney, p. ex.); type d'action dont il s'agit (les [titres] aurifères, p. ex.); devises; indices boursiers (le Dow Jones); etc. Il nous a semblé souhaitable de regrouper tous ces éléments, compte tenu de la combinatoire tout à fait stéréotypée qui les caractérise. 


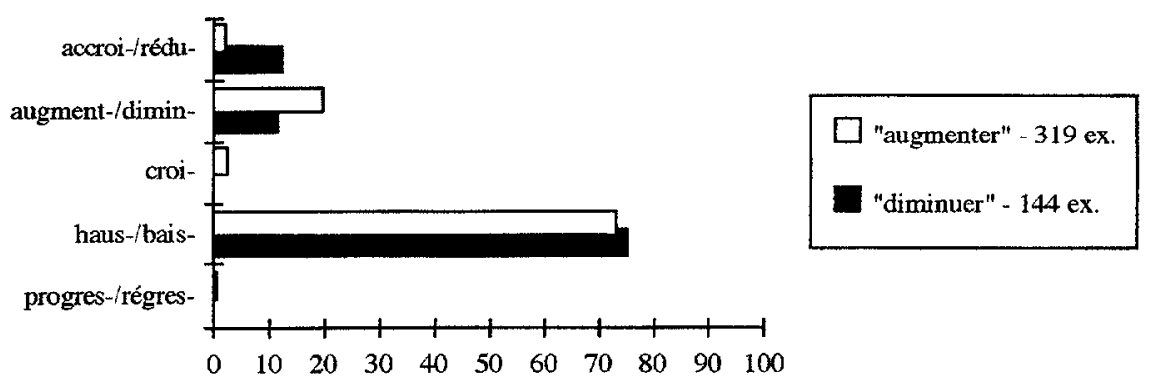

Graphique 6:

Combinaison VF et prix

Une image assez similaire se dégage des graphiques 5 et 6 avec une prépondérance d'emploi des couples haus- (les mots hausse et hausser) et bais- (les mots baisse et baisser). Cette combinaison préférentielle se retrouve également, mais de façon moins prononcée, pour les mots salaire, bénéfice, chiffre d'affaires, etc., qui partagent avec prix un trait sémantique hypothétique: «somme d'argent». Une distribution analogue se rencontre également pour le mot taux.

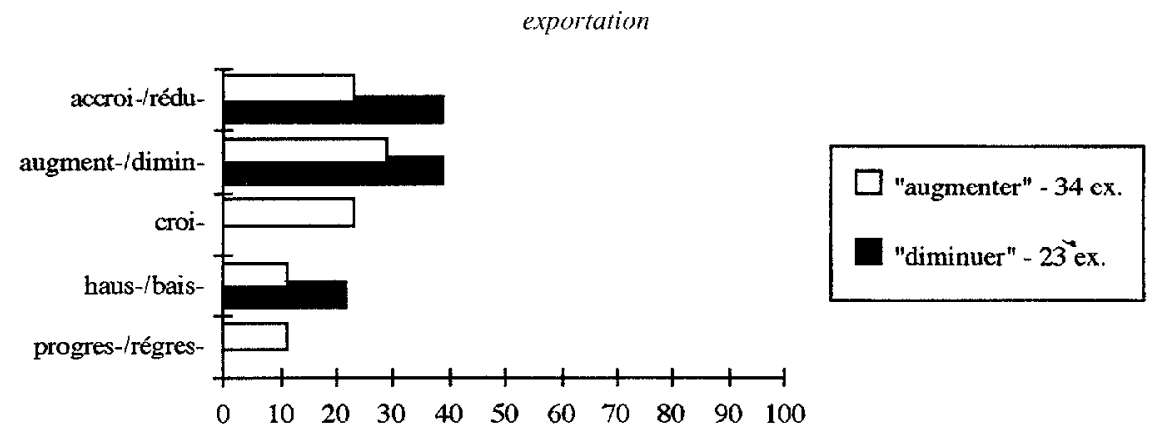

Graphique 7:

Combinaison VF et exportation 
Le type de combinatoire propre à exportation se retrouve pour la plus grande partie du vocabulaire économique. L'aspect de la combinatoire diffère de celui des graphiques 5 et 6 puisque la prépondérance des couples haus-/bais- a complètement disparu au profit des couples accroi-/rédu- et augment-/dimin-.

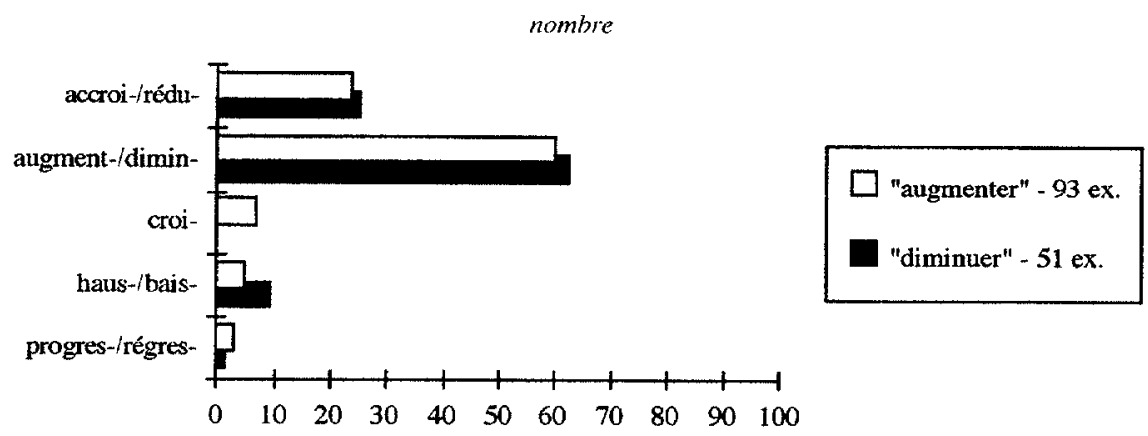

Graphique 8:

Combinaison VF et nombre

Les mots nombre, ainsi que part, volume, etc., ne sont pas à proprement parler des termes économiques, mais ils appartiennent au vocabulaire général d'orientation scientifique (VGOS, Phal 1970). Ces mots sont très fréquents dans les textes économiques en combinaison avec le VF. La dominance des couples augment-/dimin- et accroi-/ rédu-, analogue à celle relevée pour exportation, est tout à fait caractéristique pour cet ensemble de mots.

Certains termes économiques développent une combinatoire qui s'écarte de ces tendances générales. Ce fait semble dû parfois à certaines combinaisons sémantiques particulières. Tel est le cas pour la notion PIB dont la combinaison extrêmement fréquente avec le substantif du VF croissance (plus de $70 \%$ des cas) est synonyme du sens «croissance économique (d'un pays)» propre à croissance dans son emploi absolu. Tel n'est pas le cas, croyons-nous, pour la combinaison quasi obligatoire (plus de $80 \%$ des cas) de dépense avec le couple rédu-5.

\section{DU CORPUS À LA LEXICOGRAPHIE}

Comme nous l'avons fait remarquer dans notre introduction, le problème de la combinatoire du VF s'était posé dans le cadre d'une description lexicographique, le DICOFE. En fonction des données recueillies, il convient d'en présenter un traitement adéquat dans la perspective de ce dictionnaire.

Dans le texte du DICOFE, nous n'avons repris explicitement que les combinaisons à forte tendance préférenticlle :

le cours est en hausse/baisse à la hausse / baisse une hausse / baisse des prix

une hausse / baisse des salaires les salaires augmentent
(Verlinde et al. 1995: 75)

(Verlinde et al. 1993b : 55)

(Verlinde et al. 1995 : 57)

(Verlinde et al. 1995: 11) 
Il ne nous paraît pas nécessaire d'aller plus loin dans un dictionnaire qui s'adresse à des apprenants d'un niveau intermédiaire. La plupart des autres combinaisons restent en effet possibles puisqu'elles sont attestées. Le contenu du message passera donc: tout au plus certaines combinaisons peu fréquentes surprendront-elles un peu le locuteur natif.

Par contre, dans un ouvrage de référence consacré au français économique (ou des affaires) destiné à la fois à des locuteurs natifs et à des apprenants, ces informations ont leur place.

Ainsi, afin de tirer pleinement profit des informations recueillies, il nous semble nécessaire d'en arriver à une description dictionnairique qui dépasse le cadre exigu d'une entrée isolée pour chaque mot au profit d'une entrée FLUCTUATIONS consacrée à l'ensemble du phénomène. Cette entrée devrait fournir en premier lieu un inventaire de termes, classés selon leur sens, sur le modèle du Longman Language Activator (1993). Un groupe de termes correspondrait au sens «devenir/rendre plus grand, plus important»; un autre au sens «devenir moins grand, moins important». À l'intérieur de ces deux groupes, les informations pourraient être disposées selon le modèle proposé dans le Dictionnaire explicatif et combinatoire du français contemporain (Mel'čuk et al. 1984, 1988 et 1992): indications sur les constructions syntagmatiques possibles de chaque terme (voir Verlinde 1995, section 7) et sur leur combinatoire, sans toutefois reprendre la formalisation du Dictionnaire explicatif et combinatoire afin de rendre la description plus accessible à des apprenants.

On remarquera qu'en ce qui concerne les informations de type syntagmatique, il convient, dans la perspective de faciliter l'encodage de l'étudiant, de présenter non seulement la syntaxe verbale, mais également la syntaxe nominale (voir, par exemple, Mel'čuk et al. 1984, 1988 et 1992).

Comme nous l'avons vu à partir des graphiques 5 à 8 , certains types de combinatoire semblent caractéristiques de classes de mots, bien que nous ayons à garder quelques réserves à ce sujet. Les tendances que nous proposons dans ce qui suit sont donc à confirmer par le biais d'une étude plus vaste encore.

Pour les termes économiques, nous avons:

1. le vocabulaire de la bourse

un mot qui désigne une somme d'argent (prix, salaire, etc.) + haus-/baisle mot taux

Remarque: les verbes hausser et baisser s'emploient peu au profit de progresser ou monter (pour hausser) et céder (pour baisser)

2. PIB, PNB + croi- (par analogie avec croissance économique)

3. autres notions économiques :

a au sens «augmenter»: combinaison avec augment-, accroi- et, dans une moindre mesure, avec croi-;

- au sens «diminuer» : combinaison avec rédu- et, dans une moindre mesure, avec dimin-.

Remarque. Le couple rédu-semble surtout se combiner très fréquemment avec des termes à connotation négative : dépense, impôt, déficit. Cette tendance n'est cependant pas applicable systématiquement: un mot comme chômage constitue par exemple une exception.

Pour le VGOS, le type de combinatoire suivant a été relevé :

1. une indication de quantité (nombre, part, volume) + augment-/dimin(dans une moindre mesure accroi-/rédu-). 
L'article pourrait être complété par des informations non pertinentes pour l'encodage, telles que la prépondérance de l'emploi des substantifs hausse, baisse et croissance sur leurs verbes afférents et des verbes accroître, augmenter, diminuer, progresser, réduire et régresser pour les autres couples de mots.

Une telle description gagne sans conteste en qualité scientifique et pédagogique par le traitement simultané de termes apparentés. Cela la rend utilisable non seulement pour le décodage d'un message, mais aussi pour son encodage: indications précises sur le contexte d'emploi et constructions dans lesquelles entrent les termes.

\section{CONCLUSION}

En marge des implications pratiques de notre étude pour les descriptions lexicographiques, il convient de formuler quelques remarques d'ordre plus général. Ainsi, la question se pose de savoir si nous assistons à la codification progressive de certaines combinaisons particulièrement fréquentes (vocabulaire de la bourse + haus-/bais-, par exemple). On notera par ailleurs que la plupart de ces combinaisons se rencontrent avec des termes économiques à haute fréquence dans le corpus. Elles sont plus floues dès que la fréquence des termes économiques baisse. Nous rejoignons ainsi les remarques formulées par Danell (1995) dans une étude du phénomène de la concurrence lexicale en français moderne. Il affirme que ce sont précisément les structures et les expressions un peu fréquentes qui entrent dans la compétence du locuteur (Danell 1995: 18), alors que celuici improvise lorsqu'il est confronté à un besoin d'expression «insolite», peu fréquent (Danell 1995: 122), ce qui expliquerait la variété des combinaisons relevées pour les termes économiques moins fréquents.

Il serait également intéressant d'examiner si des tendances analogues se révèlent pour d'autres langues.

La synonymie des mots étudiés semble toute relative. Elle peut être maintenue du point de vue du sens, dans la perspective du décodage d'un message. Par contre, elle ne vaut plus (ou en tout cas, elle vaut moins) sur le plan de la combinatoire, dans la perspective de l'encodage d'un message, où deux groupes de mots semblent s'opposer: haus / bais- face à augment-/dimin- et accroi-/rédu-. De ce point de vue, il serait intéressant d'analyser de plus près les nombreux synonymes répertoriés sous des fonctions lexicales identiques dans Mel'čuk (1984, 1988 et 1992), afin de découvrir si l'on retrouve dans d'autres portions du vocabulaire des combinaisons préférentielles analogues à celles que nous avons découvertes pour le vocabulaire des fluctuations.

\section{Notes}

* Cet article est issu d'une communication présentée par l'auteur aux IV ${ }^{\mathrm{cs}}$ Journées scientifiques du réseau «Lexicologie, terminologie, traduction» de l'AUPELF-UREF (Lyon, France, 28, 29, 30 septembre 1995).

1. Trois tomes thématiques, consacrés respectivement à l'entreprise, au commerce et au monde de la finance, sont parus à l'heure actuelle (Verlinde et al. 1993a, 1993b ct 1995). Un quatrième, consacré à l'emploi, est en préparation.

2. Voir Verlinde et Binon (1994) pour une présentation détaillée du DICOFE.

3. On trouvera une description plus détaillée de notre étude dans Verlinde (1995).

4. Dans ces articles figurent entre autres les articles «économie» du corpus CALIBSO, qui nous a été aimàblement cédé par K. J. Danell (voir Danell 1990 pour une description détaillée de ce corpus).

5. Pour davantage de donnés chiffrées et visualisées, nous renvoyons à Verlinde (1995), section 5.2 . 


\section{RÉFÉRENCES}

DANELL, K. J. (1990): «Corpus de journaux francophones sur ordinateur», Travaux de linguistique, 20, pp. 73-82.

DANELL, K. J. (1995) : Le phénomène de concurrence en français moderne. Réflexions à partir de an-année, jour-journée, paraître-apparaître, et d'autres, Uppsala, Swedish science press, $128 \mathrm{p}$.

HERREMANS, J. (s.d.) : L'économie belge. Une analyse des principales activités économiques, s.l.

Le Grand Robert de la langue fransaise (1985): Paris, Le Robert.

Longman Language Activator (1993): Londres, Longman.

MEL'ČUK, I. A. (1984, 1988, 1992): Dictionnaire explicatif et combinatoire du français contemporain, $I, I I$, III, Montréal, Presses de l'Université de Montréal,

PHAL, M.A. (1970) : «Le vocabulaire général d'orientation scientifique : essai de définition et méthode d'enquête», J.-L. Descamps et M. Hamon, Les langues de spécialité. Analyse linguistique et recherche pédagogique, Actes du Stage de Saint-Cloud (23-30 novembre 1967), Strasbourg, AIDELA, pp. 94-1 15.

Trésor de la langue française (1971-1994): Paris, éd. du CNRS et Gallimard.

VERLINDE, S. (1995) : «La combinatoire du vocabulaire des fluctuations dans le discours économique», Cahiers de lexicologie, 66, pp. 27-66.

VERLINDE, S, et J. BINON (1994) : «The Dictionnaire contextuel du français économique: a production oriented dictionary of Business French», W. Martin (Ed.), Euralex 1994 Proceedings. Paper submitted to the oth EURALEX International Congress on Lexicography in Amsterdam, The Netherlands, Amsterdam, VU, pp. 523-529.

VERLINDE, S., BINON, J. et J. VAN DYCK (1993a) : Dictionnaire contextuel du français économique. Tome A : L'entreprise, Leuven, Garant, $80 \mathrm{p}$.

VERLINDE, S., FOLON, J., BINON, J. et J. VAN DYCK (1993b) : Dictionnaire contextuel du franşais économique. Tome B: Le commerce, Leuven, Garant, $96 \mathrm{p}$.

VERLINDE, S., FOLON, J., BINON, J. et J. VAN DYCK (1993b) : Dictionnaire contextuel du français économique. Tome $C$ : Les finances, Leuven, Garant, $118 \mathrm{p}$. 